\title{
Electronic Health Record in Dermatology Service
}

\author{
Duarte Júlio $^{1}$, Portela Filipe ${ }^{2}$, Santos Manuel $^{2}$, Abelha António ${ }^{1}$, Machado José ${ }^{1}$ \\ ${ }^{1}$ Universidade do Minho, CCTC - Braga, Portugal \\ ${ }^{2}$ Universidade do Minho, ALGORITIMI - Guimarães, Portugal \\ *Email: ${ }^{1}\{$ jduarte, abelha, jmac $\} @$ di.uminho.pt, ${ }^{2}\{\mathrm{cfp}, \mathrm{mfs}\} @$ dsi.uminho.pt
}

\begin{abstract}
In this paper we describe the implementation of an Electronic Health Record in the Dermatology service of a Portuguese hospital. This system must follow the principle of simplicity, enabling recording quality and analytical processing. Standards and norms were also followed and it is shown that interoperability has a key role in the whole process. This project is a good example of cooperation between academic and healthcare institutions and shows the impact of new technology on healthcare organizations.
\end{abstract}

Keywords: Health Records, Interoperability, XML.

\section{Introduction}

Biomedical Informatics (BMI) is a multi-disciplinary area, which results from two main disciplines: Information Technology (IT) and the area of Medicine and Health Sciences The BMI is an issue of growing importance worldwide. The contribution can provide the modernization and improvement of the quality of delivering health services through better management of health information resources [1]. In the context of the need for management and organization of information, Hospital Information Systems (HIS) are arising. HIS can be defined as a subsystem hospital with a socio-technological development, which covers all information processing as well as the role of clinicians [2]. Its main purpose is to contribute to the quality and efficiency of healthcare. This objective is primarily oriented to the patient after being directed to health professionals as well as the functions of management and administration [3]. It is very important that a HIS is designed and implemented efficiently especially when it concerns the high quality of healthcare [3]. This implementation requires the existence of a management structure whose specific function focuses on the proper allocation of resources in order to ensure the efficient production of information [4]. Thus, the planning of HIS will cover, in a systematic way, each of the components in order to provide the complex clinical decision making capacity. In fact, if requirements of appropriate access to relevant data are not met, it 
becomes difficult to take decisions, diagnoses and other procedures resulting in fatal consequences for the patient. The HIS also assumes much importance in relation to costs since the sector of communication technologies in healthcare is increasingly important [3]. There are four basic functional processes, which the most important is healthcare itself. This process list begins with the patient's admission and ends in discharge or transfer to another institution. The other categories will serve to support the healthcare with the primary objective of improving quality. The four functional categories are characterized as follows: care; clinical process management; work organization and resource planning; and hospital management $[5,6]$.

The Implementation of this project's main objective was to provide a record with quality for the Clinical Dermatology Service in Centro Hospitalar do Porto, one of the major Portuguese hospital centre. This form of registration would have to take into account various factors including some that are general to any clinical records and other more specific to the dermatology. The registration form will need to ensure that it is visible and accessible to any clinician in order to solve the problem of misinterpretation of clinical information and promoting interoperability between people and systems.

\section{Electronic Health Record}

Electronic Health Record (EHR) is assumed as a HIS for excellence and has replaced the traditional manual recording in Paper Clinical Process (PCP). EHR may include all hospital areas with a need for registration information. This information can be clinical, administrative and financial $[7,8]$.

There are several definitions for EHR but in general this can be defined as the computerized records of patients' clinical data i.e. all information provided by demographic data, medical history, laboratory data, and more information sources. These data are inserted in an electronic system that enables the capture, maintenance, transmission and storage of clinical information which is essential, not only for the monitoring of the health status of each patient but also for purposes such as cost management $[7,9,10]$. Historically, first systems appeared in the 60's giving the first impulse of EHR in history, Although these early systems are not yet focused specifically on the clinical $[11,12]$. In Portugal, the first breakthrough in the electronic record hospital came up with a system called SONHO. This system is geared to managing only administrative information. Currently, there are already systems in clinical information management the Medical Support System (SAM) and Support System for Nursing Practice (SAPE). There are also several implementations of EHR in Portugal but still with little dissemination [7].

\subsection{EHR functionalities}

In order to facilitate and improve care in health establishments, the EHR performs some features, some of which are similar to the PCP. These features include: to maintain a history of each patient serving as a support assistance for the purpose of 
decision support or as a source of information for clinicians; to reduce the frequency of loss of records or record data as well as to reduce the occurrence of medical errors; to support communication between external sources of medical information, management and resource planning; to improve procedures for assessing the cost control. The main difference with operation of the EHR and the PCP is related to the interaction between several heterogeneous information sources. The linkage of information sources and the EHR is bidirectional; i.e. there is a feedback that does not exist for the PCP. Thus an electronic record in addition to containing the information set also allows the sharing of information among different users, as well as the interaction between information sources [9].

\subsection{EHR requirements}

The success of EHR implementation depends on a set of requirements that must be considered. In hospital environment, the EHR have many common features with PCP but it should have a response, which must be fast, reliable and safe, It should be also accessible 24 hours a day. The structure of the EHR must allow seamless integration with existing HIS by promoting the ubiquity of records between different specialties and services. The ubiquity of the EHR will allow access to mechanisms for monitoring alarm systems and decision support. The electronic record will allow generation of documents and customized reports for specific purposes. It will become easier to configure interfaces for registration and more. The information contained herein must be standardized and uniform, and health professionals must be targeted by specific training $[7,13,14]$.

\subsection{EHR advantages}

Comparing with the PCP, the most advantage of the physical EHR is a small footprint device capable of storing an enormous amount of information. This advantage may still become more accentuated with the development of computer science [7, 15]. Another positive point is the duplication and sharing of information. This task becomes simpler and more immediate. This advantage enables the sharing of Clinical Process (PC) for different task synchronous implementation of clinical files to backup information and safeguarded the chance of loss and/or damage, which often happens with the PCP [7, 15]. Analysing the advantages at a structural level, the electronic record supports customizing the user interface, allowing the use of different layouts of insertion and viewing information under the very useful aspect of the availability of specific modalities in the hospital. The EHR also ensures the readability of data (it does not happen with the PCP, since the data are entered manually and their legibility depends on the handwriting of health professionals). At this level, another important advantage is that the EHR can perform the processing of continuous data, facilitating the detection of errors and issuing alarms in situations of possible pathologic abnormality. Finally, the EHR enables the automated collection of clinical parameters from monitors, imaging equipment, chemical analysis, among others [7, 15]. 


\section{AIDA}

AIDA (Agency for Integration, Diffusion and Archive of Medical Information) is a platform that consists of a Multi-Agent System (MAS) and overcomes difficulties in achieving uniformity of clinical systems, as well as medical and administrative complexity of different Hospital information sources [16]. AIDA was created by a group of researchers from the University of Minho, the Artificial Intelligence Group, and is currently installed at the Centro Hospitalar do Porto, Centro Hospitalar do Alto Ave, Centro Hospitalar do Tâmega e Sousa and Unidade Local de Saúde do Alto Alentejo. It is a good example of the successful cooperation between the University and hospitals. It is an electronic platform that provides employees with intelligence, the agents. This platform features a pro-active behaviour in its main functions: communication between heterogeneous systems, storage management and hospital information; response to requests in time; sending and receiving information from hospital sources like laboratories (labs) (medical reports, images, prescriptions, etc.). Thus, AIDA enables interoperability between hospital subsystems, assuming a main role where it is installed, as shown in figure $1[17,18]$. It can be seen in Figure 1 that AIDA has an easy access for your users, allowing the management of clinical information anywhere in the hospital. In addition, the platform enables the sending of messages via phone or e-mail. The same way, AIDA establishes connection with all Systems of medical information: EHR; Administrative Information System (AIS); Medical Information System (MIS); and Nursing Information System (NIS) [18].

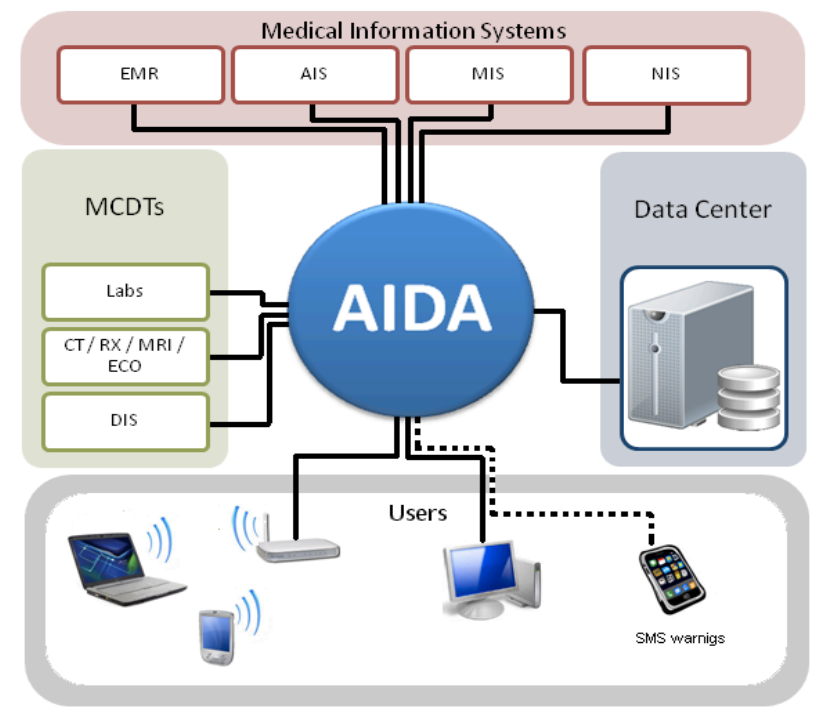

Fig. 1. HIS Structure. 


\section{AIDA-PCE}

The AIDA-PCE is a EHR and was implemented in the Centro Hospitalar do Porto. It is a subsystem of the HIS. The AIDA-PCE follows a problem-oriented organization suggested by Lawrence Weed in the 60's. This information organization is known as the Problem Oriented Medical Record (POMR) and it assumes that registration is a production of clinical scientific document. In this type of organization, clinical information (annotations, therapeutic, diagnostic) should be recorded for specific problem solving, creating a list of issues organized in a tree structure, where each new problem derives from the main branch [7, 10, 19]. One's note that problems can be classified as active or inactive, in which active problems are those where the disease is still active or even when intervention is required immediately. On the other hand, inactive problems require no urgent action. In AIDA-PCE problems assets are monitored and recorded daily using a SOAP (Subjective, Objective, Assessment and Planning) framework. Thus, each record contains the patient's symptoms, a doctor's observation, an analysis of diagnosis and a treatment plan that the patient is subject to $[7,19]$. Figure 2 shows how the registration of clinical information is performed in AIDA-PCE. Looking at the figure, it appears that all the episodes begin with an admission form and finish with an outcome. An episode is the set of all operations for the patient, since the start of treatment until the end. Each episode is built upon an Integral Database (BDI), the Problem List, the Therapeutic Plan and subsequent records. It is noted that this register can be updated during the episode and along the evolution of the patient.

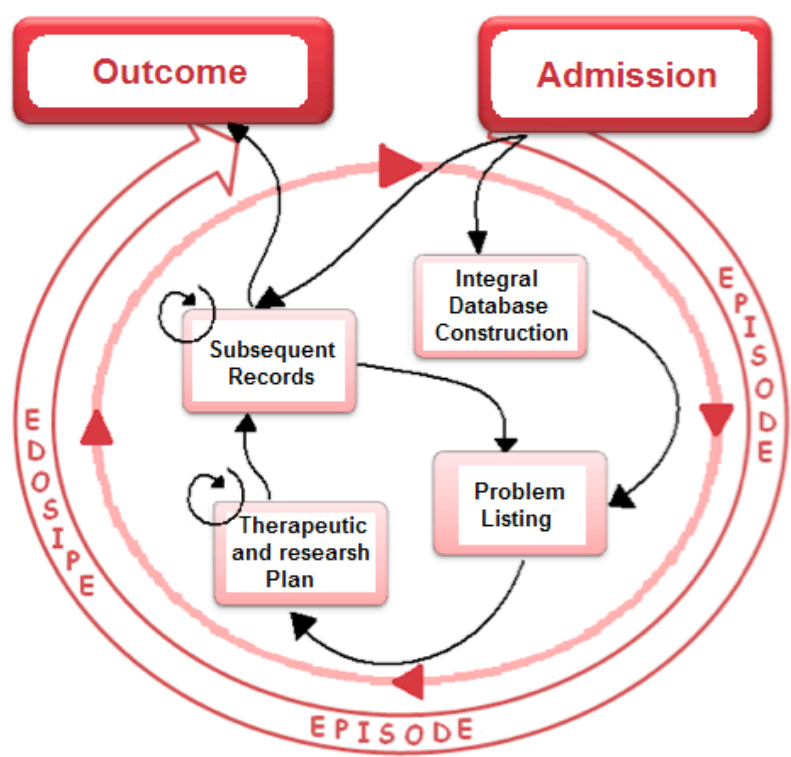

Fig. 2. HIS Structure. 


\section{Normalization}

One important property of AIDA-PCE is its interaction with different systems and the communication with biomedical devices. The information to transfer must be standardized and normalized. It is necessary that all data to be structured and misinterpretation to be forbidden. We must also take into account the database semantics, so information can be understood by different systems. In addition, the use of standards in the AIDA-PCE ensures the best communication between health professionals and interoperability between systems, allowing some automation in the hospital recording. The standards used in EHR are divided into three different purposes: standards for representing clinical information; communication standards; and image standards [7]. International Classification of Diseases, Ninth Revision, Clinical Modification (ICD-9-CM), Systematized Nomenclature of Medicine-Clinical Terminology (SNOMED-CT) and International Classification for Nursing Practice (ICNP) are standards for classification of diseases and therapeutic clinics, where each therapy or disease is associated with a code recognized anywhere in the world. The use of these standards ensures that the EHR can be readable by any clinician in the world, also allows machines to interpret symptoms and can assist clinicians in making a diagnosis and treatment plan decision [7, 20]. As communication standards, the AIDA-PCE uses the Health Level Seven (HL7) as a protocol for exchanging messages, and web architectures and service-oriented architectures (SOA). HL7 protocol was created in order to solve the problems in the exchange of messages in the institutions providing medical care. In general, the HL7 defines an organized structure for the exchange of information, ensuring easy understanding of information by the recipient. This protocol also specifies that these messages are created and shared in extensible Markup Language (XML). This language has the peculiarity of being able to separate content and presentation format of the same information, thus, the same information can be accessed in different presentation formats and different media (computer monitor, PDA, phone, etc.) [7, 21]. SOA provides easy access for software specific functions and procedures.

\section{Implementation}

The big purpose of this project was to parameterize, structure and standardize the data to be inserted in the PCE. It was used the Service of Dermatology of the Centro Hospitalar do Porto as a service test. Several forms in XML were developed, to be used to insert, consult and manage data.

\subsection{Implementation Process}

The deployment followed an iterative process based on four phases: Information Gathering and Specification; Implementation; Testing; and Training. Previous literature searches and subsequent meetings with clinicians in the Department of Dermatology created the first phase, called Data Collection and Specification. 
Research led to the vocabulary knowledge and the general functioning of a dermatology department. The meetings with the physicians responsible guaranteed the collection of information on practices and methods used in this hospital service. The Implementation phase, or codification, was based on the digitization of all information collected by this stage, thus creating the forms in XML. The next phase, called testing phase, has proved to be of great importance to the whole process. At this stage, all forms were submitted to various tests, at a computer level, and testing of clinical level, where it was evaluated the functionality of the forms under a health professional point of view. The first tests were carried out by a form designer and served to correct function errors. The next tests were performed in the presence of medical professionals involved in the project. The latter tests allowed significant corrections at the level of form performance in hospital real time, thereby generating a mini-cycle between the second and third stages. After approving, it was performed the last stage of the process, called the Training. This phase consisted of training and presentation of the new registration method to all clinicians in the Dermatology Service. Figure 3 shows the implementation process adopted in this project.

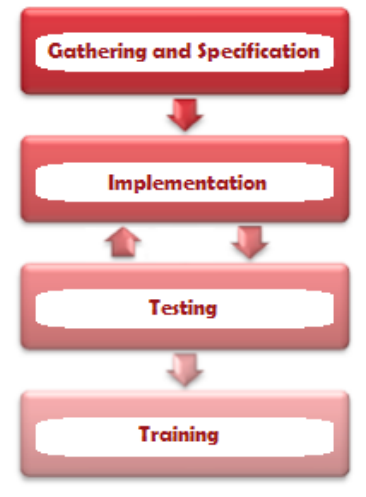

Fig. 3. Phases.

\subsection{Concerns in the Construction of Forms}

A major concern of the implementation team was the constant insistences of monitoring health professionals across the implementation process. The routines used everyday by clinicians cannot be changed too much. In the same way, this monitoring allows for a greater familiarity between clinicians and the electronic record. So each form contains only useful information to clinicians in the specific task it performs. Another big concern of the project was the standardization of clinical terms, eliminating ambiguity in information exchange. The list of parameterized data was based on ICD9, system adopted by the Portuguese government. Two more concerns taken during the construction of the forms were the largest possible parameterization 
of the data, reducing the free text fields, and greater use of combo boxes instead of checklists. The greatest possible reduction of free text fields reduces the time spent by clinicians in completing the forms and facilitates further data analysis for comparative studies and statistical data. This preference proved to be important, since the service in question produces annual control statistics. Combo boxes preference is justified by the fact that the checklists have several interpretations to the clinician. With combo boxes one and only one selection is made.

\subsection{Structure Forms}

The structure of forms can be divided into three distinct parts. The first part contains patient reference and admission information; such as reasons, history of the disease, personal and family history, among others. The second part of the structure can be called the body of the form. This part contains more specific information of each service involved. In the case of this pilot service, there is greater information on the dermatology department. In this project we can find a third part that is related to the destination for the patient at the end of the medical act. Here are fields such as plan, destination, next appointment, among others.

\subsection{Inteoperability}

The forms follow other characteristics that are of great importance to facilitate and assist the work of clinicians. The forms allow to import data that has been previously saved in PCE and import data from other subsystems (SONHO, SAM, laboratories). Another particularity of the forms is the function to perform calculations and arithmetic operations (for example, to calculate the Body Mass Index (BMI)). These forms allow fro using pre-loaded texts, when the doctor prepares a report with fields filled in by default. The forms allow for assigning scores to the state in certain patient evaluations. As a rule, the clinician classifying the patient's condition in many ways and forms returns the value of the final score providing the current status of the patient. This feature was used to assess the patient's physical status.

\section{Conclusions and Future Work}

In this paper we presented a solution to adopt health records in a Dermatology service, as an pilot service. The main goals are to improve the quality of information in order to support the patient problem solving and the development of clinical research through the use of historical cases. The use of EHR is now a reality in the Centro Hospitalar do Porto, one of the Portuguese major public hospital centre, being mandatory the use of AIDA-PCE in the hospital. The project followed the principles of simplicity, using normalization and terminology.

Based on the success of this implementation, the next step will be to extend this work for all services of the Centro Hospitalar do Porto and other health institutions. 


\section{Acknowledgment}

This research was performed with the support of the Portuguese Foundation for Science and Technology, with the grant SFRH/BD/70549/2010.

\section{References}

1. Van Bemmel, J.H., Musen, M.A. (1999). Handbook of Medical Informatics. Springer-Verlag Heidelberg.

2. Haux, R., Winter, A., Ammenwerth, E. \& Brigl, B. (2004). Strategic Information Management in Hospitals: An Introduction to Hospital Information Systems. New York: Springer-Verlag.

3. Haux. (2006). R. Individualization, globalization and health - about sustainable information technologies and the aim of medical informatics. International Journal of Medical Informatics. 75:795-808.

4. Costa, R., Novais, P., Machado, J., Alberto, C. \& Neves, J. (2007). Inter-organization Cooperation for Care of the Elderly, in Integration and Innovation Orient to E-Society. Wang, W., Li, Y., Duan, Z., Yan, L., Li, H., Yang, X. (Eds). Springer-Verlag, Series: IFIP International Federation for Information Processing. ISBN: 978-0-387-75493-2.

5. Ammenwerth E., Buchauer A., Haux R. (2002). A requirements index for information processing in hospitals. Methods of Information in Medicine. 41:282-288.

6. Hübner-Bloder G., Ammenwerth E., Brigl B., Winter A.. (2005). Specification of a Reference Model for the Domain Layer of a Hospital Information System. In Connecting Medical Informatics and Bio-Informatics Section 8: Implementation \& Evaluation of Clinical Systems. 497-502, 2005.

7. Abelha, A. (2004). Multi-Agent Systems to Support Cooperative Work in Health Care Units $\mathrm{PhD}$ Thesis. Departamento de Informática, Universidade do Minho, Braga, Portugal.

8. Slee, V., Slee, D., Schmidt, J. (2000). The Endangered Medical Record: Ensuring Its Integrity in the Age of Informatics. Tringa Press k Maxwell, A Treatise on Electricity and Magnetism, 3rd ed., vol. 2. Oxford: Clarendon, 1892, pp.68-73.

9. Neves J., Santos M., Machado J., Abelha A., Allegro S., Salazar M., Electronic Health Records and Decision Support - Local and Global Perspectives, WSEAS Transactions on Biology and Biomedicine, Issue 8, Volume 5, pp. 189-198, ISSN 1109-9518, 2008.

10. Dick, R. \& Steen, E. (1991). The Computer-based Patient Record - An Essential Technology for Health Care. Washington, DC: National Academy Press.

11. Kay, S. \& Purves, I. (1996). Medical Records and Other Stories: A Narrational Framework. Methods of Information in Medicine. 35:72-87.

12. Elberg, P.B. (2004). Electronic patient records and innovation in health care services. International Journal of Medical Informatics 64:201-205, 2001. [Gurley 2004] Gurley, L., Advantages and Disadvantages of the Electronic Medical Record, American Academy of Medical Administrators.

13. International Organization for Standardization. Requirements for an Electronic Health Record Reference Architecture - ISO 18308. Geneva: International Organization for Standardization.

14. Häyrinen K, Saranto K, Nykänen P. (2008). Definition, structure, content, use and impacts of electronic health records: A review of the research literature (Review). International Journal of Medical Informatics, 77:291-304.

15. Coiera, E. (2003). Guide to Health Informatics (2nd ed). London: Hodder Arnold. 
16. Abelha, A., Machado, J., Santos, M., Allegro, S., Rua, F., Paiva, M. \& Neves, J. (2002). Agency for Integration, Diffusion and Archive of Medical Information. Proceedings of the Third IASTED International Conference - Artificial Intelligence and Applications (ISBN 088986-390-3), Malaga, Spain, 2002.

17. Abelha, A., Santos, M., Machado, J. \& Neves, J. (2006). Auditing Agents in the Context of a Telemedical Information Society. Proceedings of the $2^{\text {nd }}$ International Conference on Knowledge Engineering and Decision Support, Knowledge and Decision Technologies. Vale, Z., Ramos, C. \& Faria, L. (eds), Lisbon, Portugal (ISBN: 972-8688-39-3).

18. Machado J., Abelha A., Novais P., Neves J. and Neves J., Quality of service in healthcare units, Int. J. Computer Aided Engineering and Technology, Vol. 2, No. 4, pp.436 to 449.

19. Bossen, C. (2007). Evaluation of a computerized problem-oriented medical record in a hospital department: Does it support daily clinical practice?. International Journal of Medical Informatics. 76:592-600.

20. http://www.ncbi.nlm.nih.gov/. Accessed in December de 2010.

21. http://www.xml.com/. Accessed in July 2010 